[Article]

\title{
重离子辐照制备电池用微孔膜及其阻抗性质
}

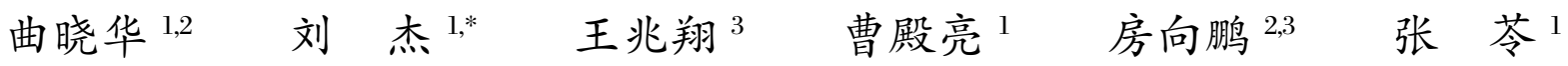 \\ 段敬来 ${ }^{1}$ 姚会军 ${ }^{1} \quad$ 陈艳峰 ${ }^{1,2} \quad$ 孙友梅 $^{1}$ 侯明东 ${ }^{1}$ \\ ( ${ }^{1}$ 中国科学院近代物理研究所, 兰州 $730000 ; 2$ 中国科学院研究生院, 北京 100049 ; \\ 3 中国科学院物理研究所, 北京 100190)
}

\begin{abstract}
摘要：利用离子辐照结合径迹蚀刻方法制备聚丙烯 $(\mathrm{PP})$ 微孔膜. 用加速器产生的单核能为 $11.4 \mathrm{MeV} \cdot \mathrm{u}^{-1}$ (总能 量 $2245.8 \mathrm{MeV}$ )的 ${ }^{197} \mathrm{Au}$ 离子束辐照 PP 膜, 剂量为 $1 \times 10^{8} \mathrm{ions} \cdot \mathrm{cm}^{-2}$. 辐照后 PP 膜沿离子路径产生损伤区域, 用硫 酸与重铬酸钾的混合液进行蚀刻(5-30 min), 制备出孔径为 380-1610 nm 的聚丙烯微孔膜. 对膜的表面和断面形 貌进行表征, 微孔膜的孔径大小及空间分布均匀, 孔道上下贯通, 形状近似为圆柱形. 给出了微孔膜的孔隙率理 论公式. 将制备的聚丙烯微孔膜用作锂离子电池隔膜, 用电化学阻抗谱(EIS)测定浸满电解液的微孔膜的离子电 导率, 并与商用隔膜进行比较. 分析表明辐照剂量和孔径大小均会影响膜的孔隙率和离子电导率, 选择合适的辐 照剂量和蚀刻时间, 可以制备出孔隙率和离子电导率符合应用标准的聚丙烯微孔膜.
\end{abstract}

关键词：电化学阻抗谱; 聚丙烯微孔膜; 锂离子电池隔膜; 快重离子辐照; 径迹蚀刻 中图分类号: 0647

\section{Preparation of Microporous Membranes by Swift Heavy Ion Irradiation and Impedance Characterization}

\author{
QU Xiao-Hua ${ }^{1,2} \quad$ LIU Jie $^{1, *} \quad$ WANG Zhao-Xiang ${ }^{3} \quad$ CAO Dian-Liang ${ }^{1} \quad$ FANG Xiang-Peng ${ }^{2,3}$ \\ ZHANG Ling $^{1} \quad$ DUAN Jing-Lai ${ }^{1} \quad$ YAO Hui-Jun $^{1} \quad$ CHEN Yan-Feng ${ }^{1,2}$ \\ SUN You-Mei ${ }^{1} \quad$ HOU Ming-Dong ${ }^{1}$ \\ ('Institute of Modern Physics, Chinese Academy of Sciences, Lanzhou 730000, P. R. China; \\ ${ }^{2}$ Graduate University of Chinese Academy of Sciences, Beijing 100049, P. R. China; \\ ${ }^{3}$ Institute of Physics, Chinese Academy of Sciences, Beijing 100190, P. R. China)
}

\begin{abstract}
Polypropylene (PP) microporous membranes were successfully prepared by swift heavy ion irradiation and track-etching. Polypropylene foils were irradiated with ${ }^{197} \mathrm{Au}$ ions of kinetic energy $11.4 \mathrm{MeV} \cdot \mathrm{u}^{-1}$ (total energy of $2245.8 \mathrm{MeV}$ ) and fluence $1 \times 10^{8}$ ions $\cdot \mathrm{cm}^{-2}$ at normal incidence. The damaged regions produced by the gold ions along the trajectories were etched in $\mathrm{H}_{2} \mathrm{SO}_{4}$ and $\mathrm{K}_{2} \mathrm{Cr}_{2} \mathrm{O}_{7}$ solutions leading to the formation of cylindrical pores in the membranes. The pore diameters of the PP microporous membranes increased from 380 to $1610 \mathrm{~nm}$ as the etching time increased from 5 to $30 \mathrm{~min}$. The surface and cross-section morphologies of the porous membranes were characterized by scanning electron microscopy (SEM). The micropores in the membranes were found to be cylindrical in shape, homogeneous in distribution, and equal in size. Some mathematical relations of the porosity of the PP microporous membranes were established by analytic derivation. The microporous membranes were used in lithium-ion batteries to measure their properties as separators. The electrical conductivity of the porous membrane immersed in liquid electrolyte was found to be comparable to that of commercial separators by electrochemical impedance spectroscopy (EIS). The results showed that the porosity and electrical conductivity were dependent on the ion fluence and etching
\end{abstract}

Received: January 20, 2010; Revised: March 22, 2010; Published on Web: April 26, 2010.

*Corresponding author. Email: j.liu@impcas.ac.cn; Tel: +86-931-4969334.

The project was supported by the West Light Foundation of the Chinese Academy of Sciences and National Natural Science Foundation of China (10775161, 10805062, 10975164).

中国科学院“西部之光”联合学者项目及国家自然科学基金(10775161, 10805062, 10975164)资助

C. Editorial office of Acta Physico-Chimica Sinica 
time. By adjusting these two factors, microporous membranes with good porosity and electrical conductivity were made that met the requirements for commercial use.

Key Words : Electrochemical impedance spectroscopy; Polypropylene microporous membrane; Lithium-ion battery separator; Swift heavy ion irradiation; Track-etching

用高能重离子辐照聚烯烃薄膜, 人射离子的能 量以电子能损和核能损的方式传递给路径上的靶电 子和原子, 当人射离子的电子能损大于材料产生缺 陷的电子能损阈值时, 由于高密度能量的沉积, 就会 在离子通道上形成柱状的损伤区域, 即潜径迹 ${ }^{[1]}$. 选 择合适的化学蚀刻方法对辐照后的聚烯烃薄膜进行 蚀刻, 可制备出核孔膜 ${ }^{[2]}$. 近年来, 核孔膜已经广泛 应用于膜分离、防伪技术、医药保健、电子工业、环境 科学以及纳米材料的制备与研究等众多领域 ${ }^{[3-8]}$. 其 特点是: 可以通过改变离子辐照参数来调节膜上孔 道的密度和排列方式, 通过改变径迹蚀刻速率 $\left(v_{\mathrm{t}}\right)$ 和 体蚀刻速率 $\left(v_{\mathrm{b}}\right)$ 的比值来调控孔道的形状, 通过改变 化学蚀刻时间来调控孔径的大小 ${ }^{[9]}$.

在电子产品不断发展和更新的今天, 锂离子电 池凭借其优异的性能在众多便携式电子产品中得到 广泛的应用. 同时随着人们环保意识和节能意识的 增强, 以及化石能源的日渐枯竭, 电动汽车的研究和 应用成为汽车工业的一个热点. 作为电动汽车的蓄 能装置和移动电源, 锂离子电池也备受关注 ${ }^{[10-11]}$. 因 而, 作为锂离子电池关键材料之一的隔膜材料也将 拥有极大的市场前景. 锂离子电池隔膜通常选用多 孔的聚丙烯 $(\mathrm{PP})$ 和聚乙烯 $(\mathrm{PE})$ 等材料. 传统的制备 锂离子电池隔膜的方法有熔融拉伸法(MSCS) 和热 致相分离法(TIPS). 这两种方法各有其优点和工艺 上的局限性, TIPS 可以较好地控制孔径及孔隙率, 但工艺复杂, 有污染, 成本高; 而 MSCS 无污染, 相 对成本低, 但孔径及孔隙率较难控制, 两种制备方 法的关键技术均被日本和欧美等少数国家垄断. 目 前, 中国还不具备优质隔膜的生产技术和规模化产 业. 国内锂离子电池生产企业所需的隔膜仍然依赖 进口 ${ }^{[12-15]}$.

本文从离子辐照技术制备核孔膜的方法出发, 制备出聚丙烯(PP)微孔膜. 用扫描电镜(SEM) 对微孔 膜进行表征, 并将制备的微孔膜用作锂离子电池隔 膜. 用电化学阻抗谱测定浸满电解液的隔膜的离子 电导率, 讨论了隔膜的微孔参数与孔隙率和离子电 导率的关系.

\section{1 实 验}

\section{1 微孔膜制备}

实验采用厚度分别为 $15 、 19$ 和 $25 \mu \mathrm{m}$, 直径为 $5 \mathrm{~cm}$ 的圆形聚丙烯薄膜(汕头冠华公司)进行研究. 用单核能为 $11.4 \mathrm{MeV} \cdot \mathrm{u}^{-1}$ 的 ${ }^{197} \mathrm{Au}$ 离子束辐照 $\mathrm{PP}$ 膜, 辐照剂量为 $1 \times 10^{8}$ ions $\cdot \mathrm{cm}^{-2}$. 辐照实验在德国 GSI 亥 姆霍兹重离子研究中心的直线加速器(UNILAC)上 完成. 将经过辐照的薄膜置于紫外灯(CHG-200, 北 京奥博迪光技术有限公司)下敏化, 每一面垂直照射 $24 \mathrm{~h}^{[16]}$. 由于聚丙烯薄膜具有耐酸碱腐蚀的性质, 因 此选取具有强氧化性的硫酸 $\left(\mathrm{H}_{2} \mathrm{SO}_{4}\right.$, 纯度 $95.0 \%$ $98.0 \%)$ 与重铬酸钾 $\left(\mathrm{K}_{2} \mathrm{Cr}_{2} \mathrm{O}_{7}\right.$, 纯度 $\left.\geqslant 99.8 \%\right)$ 的混合 液作为蚀刻液 ${ }^{[17-18]}$. 在蚀刻前依次用乙醇(纯度 $\geqslant$ 99.7\%)和去离子水清洗薄膜, 将清洗、风干后的薄膜 置于 $8 \mathrm{~mol} \cdot \mathrm{L}^{-1}$ 的 $\mathrm{H}_{2} \mathrm{SO}_{4}$ 和 $0.33 \mathrm{~mol} \cdot \mathrm{L}^{-1}$ 的 $\mathrm{K}_{2} \mathrm{Cr}_{2} \mathrm{O}_{7}$ 混合溶液中, 在 $80{ }^{\circ} \mathrm{C}$ 下进行蚀刻, 蚀刻完成后再依 次用去离子水和乙醇清洗样品并风干.

\section{2 样品的形貌和结构表征}

用场发射扫描电镜(FESEM, JSM-6701F，日本) 对微孔膜的表面和断面进行表征. 制备微孔膜的断 面样品时, 将微孔膜置于液氮中冷却大约 $5 \mathrm{~min}$, 取 出后立即折断, 淬断面的形貌能较完整地反映微孔 膜的断面信息. 用扫描电镜(SEM)对微孔膜的表面 样品和断面样品进行观测前, 先清洗所有待测样品, 并作喷金预处理, 以提高样品的导电能力. 而后观测 处理后的样品, 以得到膜的孔分布、孔道形状及孔径 大小等信息 ${ }^{[19]}$.

\section{3 离子电导率的测定}

用电化学交流阻抗谱(EIS)测定微孔膜的室温 离子电导率. 以清洁的金属锂片作为模拟电池的正 极和负极片, 将实验制备的微孔膜或商用隔膜 (Celgard $\left.{ }^{\circledR} 2325\right)$ 夹在两个锂片之间组装成双电极电 化学池, 在其中注人足量的浓度为 $1 \mathrm{~mol} \cdot \mathrm{L}^{-1}$ 的 $\mathrm{LiPF}_{6}$ (纯度 $\geqslant 99.9$ )/碳酸乙烯酯 $(\mathrm{EC}$, 纯度 $\geqslant 99.9 \%$ )+ 碳酸二甲酯 $(\mathrm{DMC}$, 纯度 $\geqslant 99.9 \%$ )(溶剂的体积比为 $1: 1$ )电解质溶液 (诺莱特科技(苏州)有限公司). 在充 满高纯氩气的手套箱(Mecaplex, 瑞士)中完成电池 的组装. 用IM6e 电化学工作站(Zahner, 德国)测定 
浸满电解液的微孔膜的电化学交流阻抗谱. 测试过 程中, 输人振幅为 $5 \mathrm{mV}$, 以频率范围为 $1-10^{5} \mathrm{~Hz}$ 的 正弦波信号作为微扰 ${ }^{[20]}$.

\section{2 结果与讨论}

\section{1 微孔膜的形貌}

微孔膜的表面形貌如图 1 所示. 图 1(a,b, c) 为 19 $\mu \mathrm{m}$ 厚的 $\mathrm{PP}$ 膜, 在 $80{ }^{\circ} \mathrm{C} 、 8 \mathrm{~mol} \cdot \mathrm{L}^{-1}$ 的 $\mathrm{H}_{2} \mathrm{SO}_{4}$ 和 0.33 $\mathrm{mol} \cdot \mathrm{L}^{-1}$ 的 $\mathrm{K}_{2} \mathrm{Cr}_{2} \mathrm{O}_{7}$ 的混合溶液中蚀刻 $5 \mathrm{~min}$ 后的不 同放大倍数的 SEM 照片. 从图中可以看出, 微孔膜 的平均孔径为 $(380 \pm 40) \mathrm{nm}$, 其中 $40 \mathrm{~nm}$ 为孔径的统 计标准偏差. 随着蚀刻时间的延长, 孔径增大. 经过 $10 \mathrm{~min}$ 蚀刻后, 相同厚度的 PP 膜的表面孔径达到 $(750 \pm 50) \mathrm{nm}$ (图 1(d)). 两种蚀刻时间下的微孔膜的 表面形貌都表明, 实验制备的微孔膜的孔分布均匀, 孔径大小大致相同; 孔的边缘清晰整齐, 形状接近 圆形(照片中孔的形状看似为椭圆形是由于观测过 程中扫描电镜样品台倾斜造成的).

图 2 是微孔膜的断面形貌. 图 $2(\mathrm{a}, \mathrm{b}, \mathrm{c})$ 是厚度 为 $15 \mu \mathrm{m}$, 经过不同时间蚀刻处理的微孔膜的断面 形貌. 将 $15 \mu \mathrm{m}$ 厚的 PP 膜蚀刻 $10 \mathrm{~min}$ 后, 其断面 上的孔径为 $(760 \pm 50) \mathrm{nm}$ (如图 2(a) 所示); 蚀刻 20 $\min$ 后, 其断面上的孔径为(1300 \pm 70$) \mathrm{nm}$ (如图 2(b) 所示); 蚀刻 $30 \mathrm{~min}$ 后, 其断面上的孔径为 $(1610 \pm 90)$
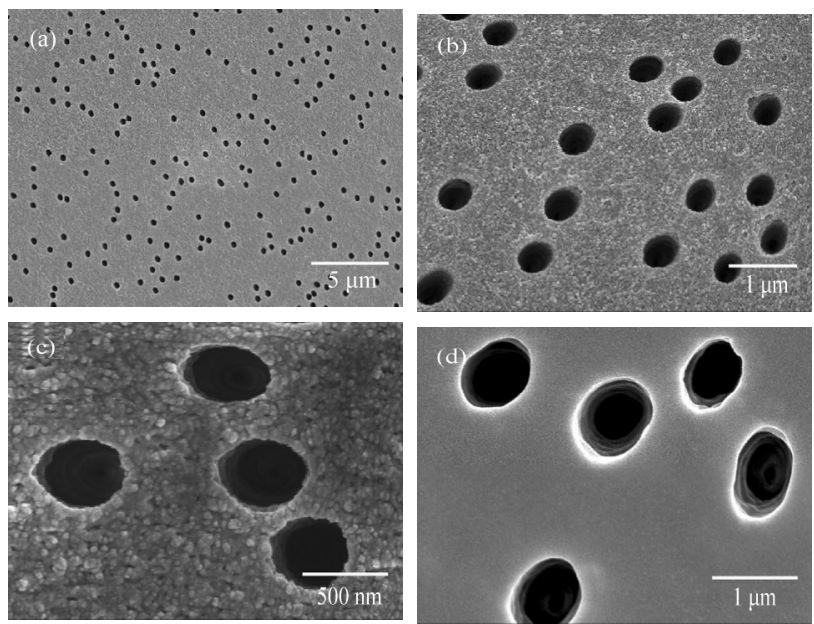

图 1 聚丙烯(PP)微孔膜的表面形貌

Fig.1 Surface morphologies of the polypropylene (PP) microporous membranes

The PP membranes with the thickness of $19 \mu \mathrm{m}$, illuminated for $24 \mathrm{~h}$ with UV light, were etched in $8 \mathrm{~mol} \cdot \mathrm{L}^{-1} \mathrm{H}_{2} \mathrm{SO}_{4}+0.33 \mathrm{~mol} \cdot \mathrm{L}^{-1} \mathrm{~K}_{2} \mathrm{Cr}_{2} \mathrm{O}_{7}$ solution at $80{ }^{\circ} \mathrm{C}$ for $5 \min (\mathrm{a}, \mathrm{b}, \mathrm{c})$ and $10 \mathrm{~min}(\mathrm{~d})$.

$\mathrm{nm}$ (如图 2(c)所示). 图 2(d)是厚度为 $19 \mu \mathrm{m}$ 的 PP 膜 蚀刻 $15 \mathrm{~min}$ 后的断面 SEM 照片, 其孔径为(1170 $60) \mathrm{nm}$. 图 2(e)是厚度为 $25 \mu \mathrm{m}$ 的 PP 膜蚀刻 $20 \mathrm{~min}$ 后的断面 SEM 照片, 其孔径为 $(1340 \pm 70) \mathrm{nm}$. 由图 $2(a, b, c)$ 可知, 厚度一定的薄膜, 随着蚀刻时间的增 加, 孔径增大.图 2 各样品的孔道基本上都是上下垂 直贯通的, 孔径分布基本均匀. 这说明蚀刻剂、蚀刻
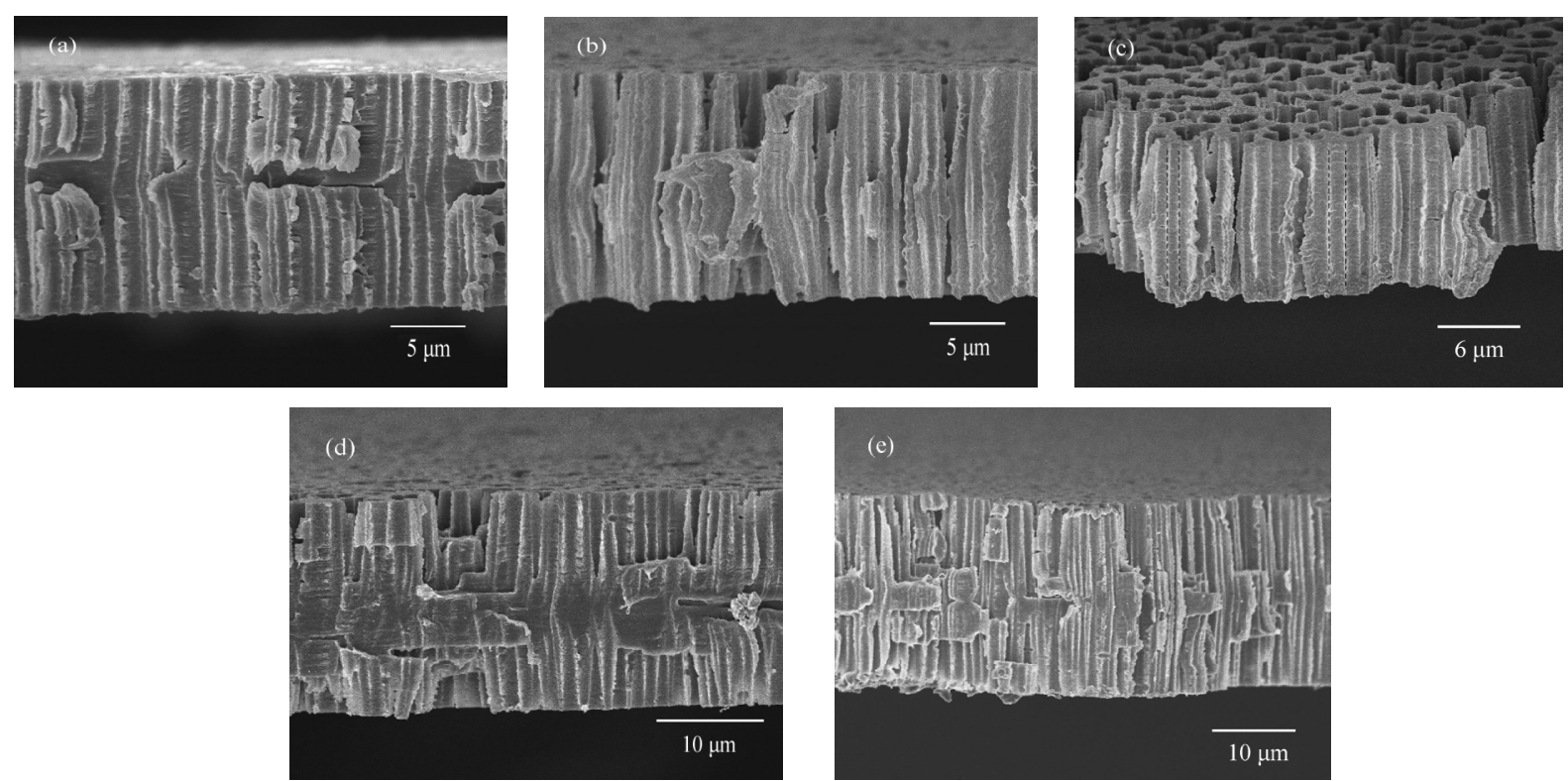

图 2 PP 微孔膜的断面形貌

Fig.2 Cross-section morphologies of the PP microporous membranes

The templates, illuminated for $24 \mathrm{~h}$ with UV light, were etched in $8 \mathrm{~mol} \cdot \mathrm{L}^{-1} \mathrm{H}_{2} \mathrm{SO}_{4}+0.33 \mathrm{~mol} \cdot \mathrm{L}^{-1} \mathrm{~K}_{2} \mathrm{Cr}_{2} \mathrm{O}_{7}$ solution at $80{ }^{\circ} \mathrm{C}$. PP film with the thickness of $15 \mu \mathrm{m}$ etched for $10 \mathrm{~min}$ (a), $20 \mathrm{~min}$ (b), $30 \mathrm{~min}$ (c); PP film with the thickness of $19 \mu \mathrm{m}$ etched for $15 \mathrm{~min}$ (d); PP film with the thickness of $25 \mu \mathrm{m}$ etched for $20 \mathrm{~min}$ (e) 
液的浓度及蚀刻时间的选择都是适当的. 微孔的内 壁整齐, 没有毛刺, 这将有利于减少电解液中阴阳离 子通过时的阻力, 提高离子电导率.

蚀刻过程实际存在两种速率: 径迹蚀刻速率 $\left(v_{t}\right)$ 和体蚀刻速率 $\left(v_{\mathrm{b}}\right)$. 对于一定厚度的薄膜, $v_{\mathrm{t}}$ 大小决 定孔的导通时间, $v_{\mathrm{t}}$ 越大则薄膜导通所需要的刻蚀 时间越短. 而 $v_{\mathrm{t}}$ 与 $v_{\mathrm{b}}$ 的比值则决定了微孔膜中孔道 的形状, 当 $v_{\mathrm{b}}$ 与 $v_{\mathrm{t}}$ 大小可比时, 孔道的形状为哑铃型 (哑铃形单孔示意图见图 3); 孔道为哑铃形的微孔膜 的孔隙率 $(\eta)^{[2] 1}$ 可由公式(1)得出.

$$
\begin{aligned}
\eta & =\frac{\pi \phi}{12}\left(3 d_{\text {in }}^{2}+3 d_{\text {in }} l \tan \theta+l^{2} \tan ^{2} \theta\right) \\
& =\frac{\pi \phi}{12}\left(3 d_{\text {out }}^{2}-3 d_{\text {out }} l \tan \theta+l^{2} \tan ^{2} \theta\right)
\end{aligned}
$$

其中 $\phi$ 为辐照剂量, $l$ 为孔道的长度 (即蚀刻后微孔 膜的厚度), $\theta$ 为孔道的倾斜角, $d_{\text {in }}$ 为哑铃形孔道的 内孔径, $d_{\text {out }}$ 为哑铃形孔道的外孔径. 当 $v_{\mathrm{t}} \gg v_{\mathrm{b}}$ 时, $\tan \theta \rightarrow 0$, 孔道的形状接近圆柱形, 孔道为圆柱形的 微孔膜的孔隙率:

$$
\eta=\frac{\pi \phi}{4} d_{\text {eff }}^{2}
$$

其中 $d_{\text {eff }}$ 为孔的平均有效孔径 ${ }^{[16]}$.

从微孔膜的断面形貌可以看出, 在上述实验条 件下对某一厚度的 PP 膜进行蚀刻时, 如果蚀刻时 间超过相应的阈值, 则膜的孔道导通. 由图 2(a,d,e) 中可以看出, 当孔道刚刚导通时, 孔道的形状略呈哑 铃形. 这是由于上述蚀刻条件近似满足 $v_{\mathrm{t}} \gg v_{\mathrm{b}}$. 当蚀 刻时间延长时, 孔道形状由哑铃形转为圆柱形, 如图 2(c)中虚线标出的两条孔道所示. 在上述实验条件 下制备的微孔膜, 其孔道接近圆柱形. 由公式(2), 孔 道为圆柱形的微孔膜, 其孔隙率与辐照剂量呈线性 关系, 与孔径大小成平方关系. 当辐照剂量一定时,

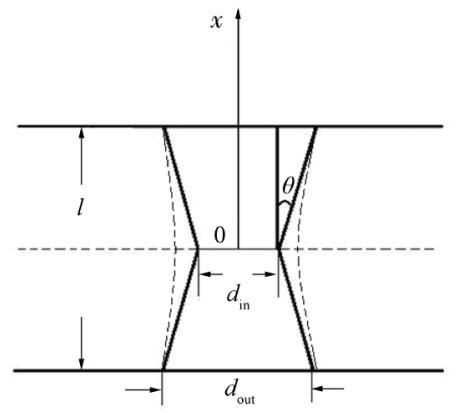

图 3 哑铃形孔道示意图

Fig.3 Schematic diagram of a double cone-shaped pore
随着蚀刻时间的增加, 孔径增大, 微孔膜的孔隙率提 高. 但当蚀刻时间过长时, 微孔膜中的孔道出现重 叠, 膜的机械强度降低. 在制备聚丙烯微孔膜时, 可 通过选择辐照剂量和蚀刻时间来调控微孔膜的孔隙 率和机械强度.

\section{2 隔膜的离子电导率}

图 4 是分别用三种不同孔径的微孔膜和商用隔 膜所组装的三明治结构电化学池的电化学阻抗谱, 其中样品 $\mathrm{a}$ 是实验制备的孔径约为 $724 \mathrm{~nm}$, 实测厚 度为 $24.27 \mu \mathrm{m}$ 的微孔膜; 样品 $\mathrm{b}$ 是孔径约为 775 $\mathrm{nm}$, 实测厚度为 $24.22 \mu \mathrm{m}$ 的微孔膜; 样品 $\mathrm{c}$ 是孔径 约为 $847 \mathrm{~nm}$, 实测厚度为 $24.15 \mu \mathrm{m}$ 的微孔膜; 样品 $\mathrm{d}$ 为实测厚度约为 $24.66 \mu \mathrm{m}$ 的商用隔膜 $\left(\mathrm{Celgard}^{\circledR}\right.$ 2325 , 聚丙烯/聚乙烯/聚丙烯 (PP/PE/PP)复合膜). 由 交流阻抗谱中圆弧与横轴的交点, 可以得到 4 种浸 润在电解质中的隔膜的本体电阻 $\left(R_{\mathrm{b}}\right)^{[20]}$. 由公式:

$\sigma=l /\left(R_{\mathrm{b}} S\right)$

可以计算出隔膜的离子电导率 $(\sigma)$. 其中 $l$ 为隔膜的 厚度, $S$ 为隔膜的面积. 图 4 的 4 个样品的相关参数 和计算结果见表 1 . 表 1 同时给出了 4 种样品的孔 隙率, 其中隔膜样品 $a 、 b 、 c$ 的孔隙率是由公式(2)计 算得出, 样品 $\mathrm{d}$ 的孔隙率参考厂家标定.

理论上, 面积为 $S$ 、辐照剂量为 $\phi$ 、微孔形状近 似为圆柱形的隔膜, 浸润电解质后的电阻 $\left(R_{\mathrm{b}}\right)$ 为

$$
R_{\mathrm{b}}=\frac{4 \rho l}{\pi d_{\text {eff }}^{2} \phi S}
$$

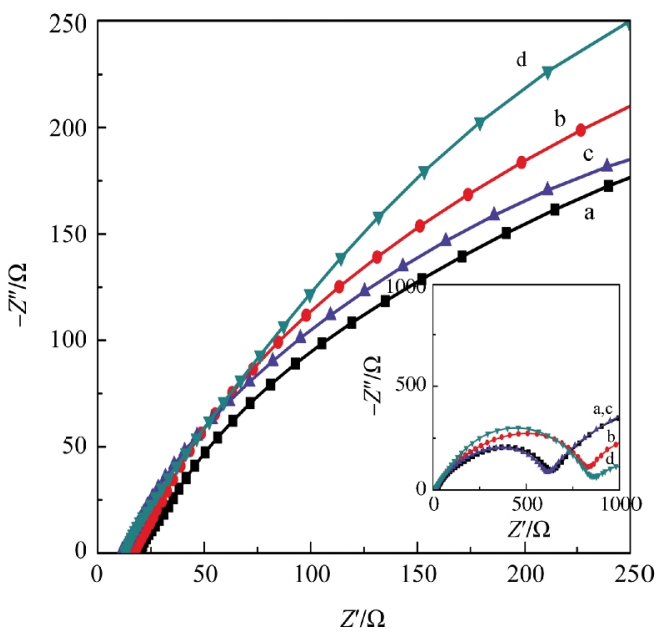

图 4 隔膜的电化学阻抗谱

Fig.4 Electrochemical impedance spectra of separators

PP separators prepared by track-etching technique with (a) $724 \mathrm{~nm}$, (b) $775 \mathrm{~nm}$, and (c) $847 \mathrm{~nm}$ diameter nanoporous;

(d) commercial separator (Celgard ${ }^{\circledR} 2325$ ) 
表 1 隔膜的相关参数和离子电导率

Table 1 Membrane parameters and ionic conductivity of separators

\begin{tabular}{cccccc}
\hline Template & $l / \mu \mathrm{m}$ & $d / \mathrm{nm}$ & $\eta(\%)$ & $R_{\mathrm{b}} / \Omega$ & $10^{5} \sigma /\left(\mathrm{S} \cdot \mathrm{cm}^{-1}\right)$ \\
\hline a & 24.27 & 724 & 41 & 19.74 & 6.49 \\
$\mathrm{~b}$ & 24.22 & 775 & 47 & 16.87 & 7.60 \\
$\mathrm{c}$ & 24.15 & 847 & 56 & 10.26 & 12.5 \\
$\mathrm{~d}$ & 24.66 & 28 & 39 & 13.04 & 9.83 \\
\hline
\end{tabular}

$l$ : thickness, $d$ : average diameter, $\eta$ : porosity, $R_{\mathrm{b}}$ : impedance, $\sigma$ : electrical conductivity; a, b, c, d are the same as those in Fig.4.

式中, $\rho$ 为电解液的电阻率, $l$ 为孔道的长度 (即微孔 膜的实测厚度). 由公式 $(3,4)$ 可知, 对于相同厚度的 微孔膜, 平均有效孔径越大, 其本体电阻越小, 离子 电导率越大.

由于制备原理不同, 离子辐照制备的微孔膜的 平均孔径远大于用熔融拉伸法制备的商用隔膜 Celgard $^{\circledR} 2325$ 的平均孔径, 且孔道结构也差别极 大. Celgard ${ }^{\circledR} 2325$ 隔膜的孔道纵横交错, 而实验制 备的微孔膜的孔道上下垂直贯通. 表 1 中 $a 、 b 、 c$ 三 种微孔膜, 随着平均孔径的增加, 膜的本体电阻逐渐 减小, 实测离子电导率依次增大, 与理论相符. 由表 1 数据可知, 实验制备的隔膜 $\mathrm{b}$ 和 $\mathrm{c}$ 的离子电导率 都接近于商用隔膜 $\mathrm{d}$ 的离子电导率, 而且隔膜 $\mathrm{c}$ 的 离子电导率略大于商用隔膜的离子电导率. 这说明, 通过改变辐照剂量和蚀刻时间, 可以调控隔膜的孔 隙率, 并能在一定范围内改变隔膜离子电导率. 因此 在上述实验条件下制备的微孔膜, 用作锂离子电池 隔膜时, 其孔隙率和离子电导率可以达到应用标准.

\section{3 结 论}

用重离子辐照技术并通过控制化学蚀刻时间制 备出了孔径为 380-1610 $\mathrm{nm}$ 的不同厚度的聚丙烯 微孔膜. 用 SEM 对膜的表面及断面进行表征, 可以 观察到微孔膜中孔道分布均匀, 孔径大小大致相同, 孔道上下贯通, 形状接近圆柱形, 孔径随着蚀刻时间 的增加而增大.

重离子辐照制备的微孔膜用作锂离子电池隔膜 时, 其孔径的大小和孔的空间分布具有很好的可控 性. 通过改变辐照剂量和蚀刻时间两个参数, 在一 定范围内, 可以人为调节隔膜的孔隙率和离子电导 率, 使其孔隙率和离子电导率达到应用标准. 但将 微孔膜用作电池隔膜的其它性能尚需要做进一步
的研究.

\section{References}

1 Hou, M. D.; Liu, J.; Zhang, Q. X. Nucl. Techniques, 2002, 25(7): 481 [侯明东, 刘 杰, 张庆祥. 核技术, 2002, 25(7): 481]

2 Apel, P. Y. Radiat. Meas., 2001, 34: 559

3 Apel, P. Y. Nucl. Instrum. Methods Phys. Res. B, 2003, 208: 11

4 Guo, H. Y.; Huang, Z. D. Nucl. Techniques, 2002, 25(7): 559 [郭洪英, 黄正德. 核技术, 2002, 25(7): 559]

5 Liu, J.; Duan, J. L.; Toimil-Molares, M. E.; Karim, S.; Cornelius, T. W.; Dobrev, D.; Yao, H. J.; Sun, Y. M.; Hou, M. D.; Mo, D.; Wang, Z. G.; Neumann, R. Nanotechnology, 2006, 17: 1922

6 Yao, H. J.; Liu, J.; Duan, J. L.; Hou, M. D.; Sun, Y. M.; Mo, D.; Chen, Y. F.; Xue, Z. H. Acta Phys. -Chim. Sin., 2007, 23(4): 489 [姚会军, 刘 杰, 段敬来, 侯明东, 孙友梅, 莫 丹, 陈艳峰, 薛智浩. 物理化学学报, 2007, 23(4): 489]

7 He, X. M.; Sun, Z. G.; Wan, C. R. Radiat. Meas., 2006, 41: 112

8 Sun, Z. G.; Zhang, Q. R.; He, X. M.; Yan, Y. S. Nucl. Techniques, 2002, 25(1): 36 [孙志国, 张泉荣, 何向明, 严玉顺. 核技术, 2002, 25(1): 36]

9 Apel, P. Y.; Blonskaya, I. V.; Dmitriev, S. N.; Orelovitch, O. L.; Sartowska, B. J. Membr. Sci., 2006, 282: 393

10 Nishi, Y. J. Power Sources, 2001, 100: 101

11 Scrosati, B. Electrochim. Acta, 2000, 45: 2461

12 Djian, D.; Alloin, F.; Martinet, S.; Lignier, H.; Sanchez, J. Y. J. Power Sources, 2007, 172: 416

13 Ihm, D. W.; Noh, J. G.; Kim, J. Y. J. Power Sources, 2002, 109: 388

14 Arora, P.; Zhang, Z. M. Chem. Rev., 2004, 104: 4419

15 Ko, J. M.; Min, B. G.; Kim, D. W.; Ryu, K. S.; Kim, K. M.; Lee, Y. G.; Chang, S. H. Electrochim. Acta, 2004, 50: 367

16 Duan, J. L.; Liu, J.; Zhu, Z. Y.; Wang, Z. G.; Sun, Y. M.; Hou, M. D.; Jin, Y. F.; Yao, H. J.; Song, Y.; Zhao, Z. M.; Yao, C. F. Nucl. Techniques, 2005, 28(9): 684 [段敬来, 刘 杰, 朱智勇, 王志光, 孙友梅, 侯明东, 金运范, 姚会军, 宋 银, 赵志明, 姚存峰. 核技 术, 2005, 28(9): 684]

17 Chakarvarti, S. K. Radiat. Meas., 2009, 44: 1085

18 Ferain, E.; Legras, R. Nucl. Instrum. Methods Phys. Res. B, 2003, 208: 115

19 Duan, J. L.; Liu, J.; Song, Y.; Yao, H. J.; Sun, Y. M.; Hou, M. D.; Mo, D.; Chen, Y. F. At. Energy Sci. Technol., 2008, 42(9): 286 [段敬来, 刘 杰, 宋 银, 姚会军, 孙友梅, 侯明东, 莫 丹, 陈艳峰. 原子能科学技术, 2008, 42(9): 286]

20 Kim, D. J. Power Sources, 2000, 87: 78

21 Pu, W. H.; He, X. M.; Wang, L.; Jiang, C. Y.; Wan, C. R. J. Membr. Sci., 2006, 272: 11 